\title{
COMMUNICATION
}

\section{Orthodontie fonctionnelle et esthétique dans un cas de parodontite agressive à l'aide de vis d'ancrage dans la pyramide nasale}

\author{
Pruvost $\mathrm{JL}^{\star}$, Andréani $\mathrm{JF}^{* *}$, Audi $\mathrm{G}^{*+*}$, Alantar $\mathrm{A}^{*+*+*}$ \\ "Cabinet d'orthodontie Kleber , Paris, France \\ " Chirurgie maxillo-faciale, Paris, France \\ "-" Centre de réhabilitation orale de l'Hôpital américain de Paris, France \\ Chirurgie orale, Paris, France
}

dr.pruvost@maxismile.org

Introduction

Les ancrages osseux sont des appuis indispensables pour mener à bien certains traitements orthodontiques notamment chez l'adulte (Pruvost et Andréani, 2013). Dans un contexte de parodontite agressive leur utilisation devient indispensable pour ne pas fragiliser les piliers postérieurs qui dans le cadre d'un recul du segment antérieur seraient invariablement sollicités au-delà de leur résistance résiduelle.

\section{Observation}

Une femme de 60 ans est atteinte d'une migration vestibulaire de son secteur incisivo-canin supérieur avec alvéolyse horizontale de $60 \%$, mobilités dentaires (3+) et gingivite avec diastèmes disgracieux. Le sondage des poches et les radiographies confirment le diagnostic de parodontite agressive. La patiente demande la suppression des « trous noirs ». Un traitement initial basé sur un enseignement à l'hygiène orale, des séances de détartrage-débridement-surfaçage et un traitement antiseptique local (chlorexidine, 0,5\%) et général (amoxicilline + acide clavulanique, $3 \mathrm{gr} / \mathrm{j}, 15 \mathrm{j}$ ) est instauré. L'indication orthodontique est posée suite à une réévaluation montrant un gain d'attache clinique significatif, une disparition de l'inflammation gingivale et une diminution des mobilités. L'objectif est de reculer le bloc antérieur. Une maintenance mensuelle est réalisée durant le traitement orthodontique. Le contrôle des mobilités fut immédiat après le placement d'un appareil lingual de type Edgewise ${ }^{\circledR}(18 \times 25)$ et l'utilisation d'un $\operatorname{arc~Niti~}^{\circledR}(.018$ inch). Le recul a pu se faire à l'aide de vis d'ancrage Deltex ${ }^{\circledR}(2 \times 13 \mathrm{~mm})$ placées de part et d'autre du raphé médian, sous anesthésie locale. Le guide chirurgical fabriqué à l'aide d'une double incidence céphalométrique de face et de profil a permis de réaliser les pré-forages $(1,3 \mathrm{~mm})$ en toute sécurité. Les vis sont surmontées d'un arc Goggle ${ }^{\circledR}$ en forme de lunette, plié à façon support de forces élastiques appliquées aux prémolaires. Le traitement a duré six mois et la contention assurée par un bridge collé et claveté. Des séances de maintenance trimestrielles sont instaurées. Le contrôle à 2 ans postorthodontie montre une enveloppe parodontale saine et une stabilité du résultat. 


\section{Discussion}

L'indication orthodontique avec une parodontite agressive, nécessite l'évaluation préalable de la gencive attachée, des poches, des mobilités, et déplacements dentaires ainsi que de l'alvéolyse. L'alternative du bridge complet sur implants implique des extractions et greffes osseuses multiples, une stratégie source de mutilations importantes mal perçues par les patients. Le préalable au traitement orthodontique est la recherche de la stabilisation de la maladie parodontale sensible à l'effet délétère des déplacements dentaires (Bolen et coll., 2008). Les forces orthodontiques doivent être légères et constantes, surtout durant la phase initiale. Les ancrages osseux rendent fiables les mécanismes d'application de forces. La diminution de la durée thérapeutique explique leur utilisation croissante (Lekovic, 2000). Les micro-vis Deltex ${ }^{\circledR}$ à double collerette, sont adaptées à l'épaisse fibro-muqueuse palatine. Leur structure autotaraudante et autocompactante facilite leur mise en place.

\section{Conclusion}

L'usage de vis d'ancrage profond dites « pyramidales » rendent possibles les traitements orthodontiques au maxillaire sur terrain parodontal amoindri. Les suites minimes de l'intervention amènent une grande adhésion des patients. Leur bonne coopération sur le plan de l'hygiène est néanmoins la clé du succès du traitement orthoparodontal. 